\title{
Papers
}

\section{DNA microsatellite instability in hyperplastic polyps, serrated adenomas, and mixed polyps: a mild mutator pathway for colorectal cancer?}

\author{
H Iino, J R Jass, L A Simms, J Young, B Leggett, Y Ajioka, H Watanabe
}

First Department of Surgery, Yamanashi Medical University, Japan

$\mathrm{H}$ Iino

Department of Pathology, University of Queensland Medical School, Herston, Queensland 4006, Australia

J R Jass

Conjoint

Gastroenterology

Laboratory, Bancroft

Centre, Royal

Brisbane Hospital,

Australia

L A Simms

J Young

B Leggett

First Department of Pathology, Niigata

University, Japan

Y Ajioka

$\mathrm{H}$ Watanabe

Correspondence to:

Professor J R Jass.

email:

j.jass@mailbox.uq.edu.au

Accepted for publication 16 October 1998

\begin{abstract}
Aim-To investigate the distribution of DNA microsatellite instability (MSI) in a series of hyperplastic polyps, serrated adenomas, and mixed polyps of the colorectum.

Methods-DNA was extracted from samples of 73 colorectal polyps comprising tubular adenomas (23), hyperplastic polyps (21), serrated adenomas (17), and mixed polyps (12). The presence of MSI was investigated at six loci: MYCL, D2S123, F13B, BAT-40, BAT-26, and c-myb T22, using polymerase chain reaction based methodology. MSI cases were classified as MSI-Low (MSI-L) and MSIHigh (MSI-H), based on the number of affected loci.

Results-The frequency of MSI increased in tubular adenomas (13\%), hyperplastic polyps $(29 \%)$, serrated adenomas $(53 \%)$, and mixed polyps $(83 \%)$ (Wilcoxon rank sum statistic, $p<0.001)$. Hyperplastic epithelium was present in nine of 12 mixed polyps and showed MSI in eight of these. MSI was mostly MSI-L. MSI-H occurred in two serrated adenomas and three mixed polyps. Clonal relations were demonstrated between hyperplastic and dysplastic epithelium in four of eight informative mixed polyps.

Conclusions-The findings support the view that hyperplastic polyps may be fundamentally neoplastic rather than hyperplastic. A proportion of hyperplastic polyps may serve as a precursor of a subset $(10 \%)$ of colorectal cancers showing the MSI-L phenotype, albeit through the intermediate step of serrated dysplasia. This represents a novel and distinct morphogenetic pathway for colorectal cancer.

(7 Clin Pathol 1999;52:5-9)
\end{abstract}

Keywords: polyp; colorectum; microsatellite instability; serrated adenoma

The adenoma-carcinoma sequence has been widely accepted as the evolutionary paradigm for colorectal cancer, and the concept has been bolstered by the recognition of molecular counterparts to the stepwise process. ${ }^{1}$ Nevertheless, it is clear that more than one morphogenetic pathway exists. For example, adenomas may be tubular or villous, flat or polypoid. K-ras mutations occur with reduced frequency in flat adenomas, a subtype believed to follow a more aggressive course. ${ }^{23} \mathrm{~A}$ more recently recognised subtype is the serrated adenoma. This combines the saw tooth or serrated configuration of the hyperplastic (metaplastic) polyp with adenomatous changes: hyperchromatism, enlargement, elongation, and pseudostratification of nuclei; deeply eosinophilic or amphophilic cytoplasm; expanded proliferative zone; dilated or horizontal crypts; and loss of mucin production. ${ }^{45}$ Serrated adenomas may, like their non-serrated counterparts, be flat or polypoid, tubular or villous. ${ }^{6}$ They have a significant malignant potential; in one series $11 \%$ contained foci of early carcinoma. ${ }^{4}$ Overexpression and mutation of p53 have been demonstrated in serrated adenomas, confirming their neoplastic nature ${ }^{7}$; p53 changes were limited to foci of high grade dysplasia or intramucosal carcinoma. ${ }^{7}$

An area of persisting controversy relates to the relation between hyperplastic polyps and serrated adenomas. Since hyperplastic polyps have traditionally been regarded as non-neoplastic, whereas serrated adenomas are neoplastic, any morphological similarities have been regarded by some as fortuitous. ${ }^{4}$ Hyperplastic polyposis has long been known to be associated with colorectal cancer, and the large serrated polyps associated with this condition have recently been equated with serrated adenomas. ${ }^{5}$ This shows that the morphological similarity of the two types of epithelial polyp is sufficiently marked to lead to diagnostic confusion. In addition serrated adenomas often occur in contiguity with typical hyperplastic polyps. ${ }^{8}$ There is now evidence suggesting that hyperplastic polyps are a form of neoplasm, because they show clonal genetic changes including K-ras mutations, DNA microsatellite instability, ${ }^{10-13}$ mutation of TGF $\beta R I I,{ }^{12}$ and rearrangements of chromosome $1 \mathrm{p} .{ }^{13}{ }^{14}$ The view that hyperplastic polyps are non-neoplastic may need to be revised, with a reappraisal of their relation with serrated adenomas. 

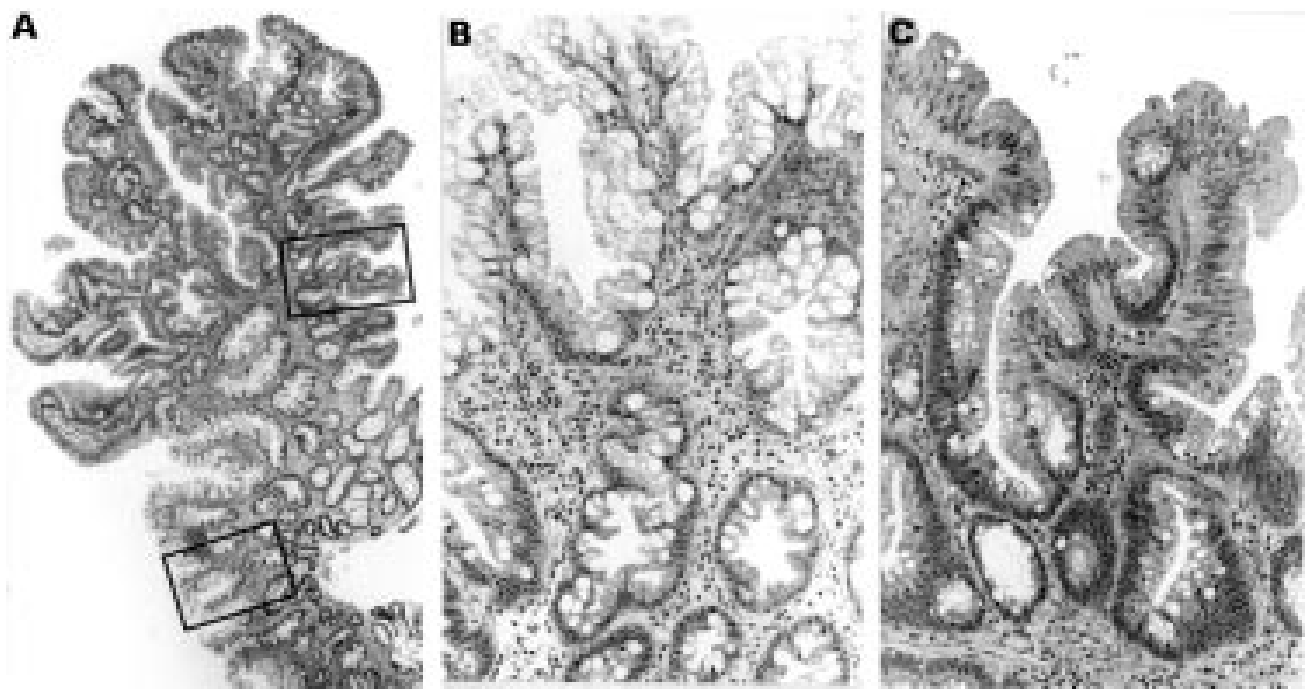

Figure 1 Mixed polyp (A) with magnified fields of hyperplastic epithelium (B) (lower box in 1A) and serrated adenoma with mild dysplasia $(C)$ (upper box in $1 \mathrm{~A}$ ). Haematoxylin and eosin.

The aim of this study was to examine the distribution of DNA microsatellite instability (MSI) in a series of hyperplastic polyps and serrated adenomas, including mixed lesions, and to determine whether these polyps might be placed in a histogenetic continuum.

\section{Methods}

COLORECTAL POLYPS AND HISTOPATHOLOGY

The material included 73 colorectal polyps comprising tubular adenomas (23), hyperplastic polyps (21), serrated adenomas (17), and mixed polyps (12). These were removed endoscopically or obtained from surgically resected specimens from 65 patients treated at Niigata University Hospital, Niigata, Japan and Royal Brisbane Hospital, Brisbane, Australia. The patients had no history of familial colorectal cancer. Fifty polyp samples were obtained from formalin fixed, paraffin embedded archival material. Serial sections were cut from each paraffin block, and the first of these was stained with haematoxylin and eosin (H\&E) for histopathological diagnosis and as a template for microdissection. Another 23 polyps, all of which were tubular adenomas, were obtained as fresh samples. Half of each bisected polyp was placed in liquid nitrogen after resection for subsequent DNA analysis. The remaining half was submitted for histological diagnosis. Histological review was made by one of us (JRJ) before DNA analysis. Precise criteria for grading serrated adenomas have not been published $^{4}$ but the polyps fell within the traditional boundaries of low grade dysplasia, apart from three mixed polyps with foci of high grade dysplasia (carcinoma in situ).

DNA EXTRACTION

DNA was extracted from tissue samples using a modified method of Levi et al..$^{15}$ Using an $\mathrm{H} \& \mathrm{E}$ stained serial section as a guide, representative areas of pure lesion or normal mucosa were gently scraped from one or two slides by a surgical blade under a dissecting microscope. Microdissection was performed with particular care in mixed polyps to prevent contamination of one subclone by another. Dissected tissues were placed in a $0.5 \mathrm{ml}$ microfuge tube with $350 \mu \mathrm{l}$ of $20 \%$ xylene and deparaffinised with xylene and ethanol. After centrifugation and drying, the pellet was resuspended in $15 \mu \mathrm{l}$ of Gene Releaser (Bio Ventures Inc). The mixture was then subjected to the following programme on a Perkin-Elmer thermal cycler: two cycles of $65^{\circ} \mathrm{C}$ for $0.5 \mathrm{~min}, 8^{\circ} \mathrm{C}$ for $0.5 \mathrm{~min}, 65^{\circ} \mathrm{C}$ for 1.5 $\min , 97^{\circ} \mathrm{C}$ for $3.0 \mathrm{~min}, 8^{\circ} \mathrm{C}$ for $1.0 \mathrm{~min}, 65^{\circ} \mathrm{C}$ for $3.0 \mathrm{~min}, 97^{\circ} \mathrm{C}$ for $1.0 \mathrm{~min}$, and $65^{\circ} \mathrm{C}$ for 1.0 min. After adding $15 \mu \mathrm{l}$ of proteinase $\mathrm{K}(0.4$ $\mathrm{mg} / \mathrm{ml}$ ), the mixture was incubated at $55^{\circ} \mathrm{C}$ for five hours and then at $95^{\circ} \mathrm{C}$ for 15 minutes to inactivate the proteinase $\mathrm{K}$. For fresh frozen samples, DNA was extracted as previously described. ${ }^{16}$

ANALYSIS OF MICROSATELLITE INSTABILITY

DNA from each pair of samples was analysed for MSI at six loci: MYCL, D2S123, F13B, ${ }^{17}$ BAT-40, BAT-26, ${ }^{18}$ and c-myb T22 (unpublished data). The reaction volume for PCR was $15 \mu \mathrm{l}$, which included $3 \mu \mathrm{l}$ of the DNA sample, $15 \mathrm{nM}$ of each primer, $3 \mathrm{mM}$ of each deoxynucleotide triphosphate, $1 \mu$ of PCR buffer, 3.75 $\mathrm{U}$ of Taq polymerase (Boehringer Mannheim), and $\left[{ }^{33} \mathrm{P}\right] \mathrm{dATP}$. The PCR reaction was run as follows: one cycle of $92^{\circ} \mathrm{C}$ for three minutes, $95^{\circ} \mathrm{C}$ for three minutes, $55^{\circ} \mathrm{C}$ for 90 seconds, 33 cycles of $92^{\circ} \mathrm{C}$ for 45 seconds, $55^{\circ} \mathrm{C}$ for 90 seconds, $72^{\circ} \mathrm{C}$ for 90 seconds, and one cycle of $92^{\circ} \mathrm{C}$ for 45 seconds, $55^{\circ} \mathrm{C}$ for 90 seconds, and $72^{\circ} \mathrm{C}$ for five minutes. After amplification, PCR products were separated by electrophoresis in 5\% denaturing polyacrylamide (19:1) gels and visualised by autoradiography. Samples which failed to amplify underwent several rounds of random hexamer PCR according to the method described by Peng et $a l^{19}$ before amplification with the test marker.

Tumours with bandshifts at one microsatellite locus were classified as MSI. These were subclassified as MSI-Low (MSI-L) when band shifts were seen at one or two of the following loci: MYCL, D2S123, F13B, and BAT-40; and as MSI-High (MSI-H) when either three loci 
Table 1 Classification of epithelial polyps

\begin{tabular}{lr}
\hline Pure polyps & 61 \\
TA & 23 \\
HP & 21 \\
SA & 17 \\
Mixed polyps & 12 \\
SA+TA & 1 \\
SA+CIS & 2 \\
HP+SA & 7 \\
HP+SA+TA & 1 \\
HP+SA+CIS & 1 \\
\hline
\end{tabular}

Pure polyps, showed homogeneous histology;

heterogeneous histological

features: CIS, carcinoma in

situ; HP, hyperplastic polyp;

SA, serrated adenoma; TA,

tubular adenoma.
Mixed polyps, showed

were affected or only two, with the proviso that one of these loci was either BAT-26 or c-myb T22. The latter are sensitive and specific for MSI-H status (unpublished observations). Scoring of MSI was undertaken independently by two of us (HI, JY)

\section{STATISTICAL ANALYSIS}

Associations between variables and MSI were assessed by $\chi^{2}$, Fisher's exact test, or Wilcoxon rank sum statistic using a Statxact package. Probability $(\mathrm{p})$ values of $<0.05$ were considered significant.

\section{Results}

Histopathological review confirmed the diagnosis of 23 tubular adenomas, 21 hyperplastic polyps, and 17 serrated adenomas with homogeneous histology. The 12 mixed polyps showed various components of hyperplastic polyp, serrated adenoma, tubular adenoma, or severe dysplasia amounting to carcinoma in situ (fig 1; table 1). DNA was extracted from the epithelial types as follows: 30 hyperplastic, 29 serrated adenoma, 25 tubular adenoma, and three foci of severe dysplasia/carcinoma in situ.

Table 2 shows the frequency of microsatellite instability. The status of MSI in each mixed polyp was determined by the epithelial type (excluding adenocarcinoma) which showed MSI at the highest number of loci. Six of 21 hyperplastic polyps (29\%), nine of 17 serrated adenomas (53\%), and 10 of 12 mixed polyps $(83 \%)$ showed MSI at one or more loci, whereas only three of 23 tubular adenomas (13\%) showed MSI. This distributional trend was significant (Wilcoxon rank sum statistic, $\mathrm{p}<0.001)$. Two of 17 serrated adenomas $(11.7 \%)$ and three of 12 mixed polyps (25\%) were MSI-H, whereas all tubular adenomas and hyperplastic polyps showing MSI were MSI-L.

In the 12 mixed polyps, two or three epithelial types were identified histologically as shown in table 1. Microdissection allowed each area to be tested separately for MSI (table 3). Eight of nine samples of hyperplastic epithe-

Table 2 Microsatellite instability in colorectal polyps

\begin{tabular}{llllll}
\hline & Total & \multicolumn{1}{c}{ MSS } & MSI-L & MSI-H & p Value* \\
\hline Tubular adenoma & 23 & $20(87.0)$ & $3(13.0)$ & $0(0)$ & \\
Hyperplastic polyp & 21 & $15(71.4)$ & $6(28.6)$ & $0(0)$ & \\
Serrated adenoma & 17 & $8(47.1)$ & $7(41.2)$ & $2(11.7)$ & \\
Mixed polyp & 12 & $2(16.7)$ & $7(58.3)$ & $3(25.0)$ & 0.001 \\
\hline
\end{tabular}

MSS (microsatellite stable), no microsatellite instability at six markers; MSI-L, microsatellite instability was seen at one or two loci of MYCL, D2S123, F13B, or BAT-40; MSI-H, microsatellite instability was positive at three loci of MYCL, D2S123, F13B, or BAT-40 or at two loci providing one was BAT-26 or c-myb.

${ }^{\star} \chi^{2}$ test. Numbers in parentheses are percentages.

Table 3 Microsatellite instability in mixed polyps

\begin{tabular}{lrlll}
\hline & Total & MSS & MSI-L & MSI-H \\
\hline Tubular adenoma & 2 & $0(0)$ & $0(0)$ & $2(100)$ \\
Hyperplastic epithelium & 9 & $1(11.1)$ & $6(66.7)$ & $2(22.2)$ \\
Serrated adenoma & 12 & $5(41.7)$ & $6(50.0)$ & $1(8.3)$ \\
Carcinoma in situ & 3 & $1(33.3)$ & $0(0)$ & $2(66.6)$
\end{tabular}

MSS (microsatellite stable), no microsatellite instability at six markers; MSI-L, microsatellite instability was seen at one or two loci of MYCL, D2S123, F13B, or BAT-40; MSI-H, microsatellite instability was positive at three loci of MYCL, D2S123, F13B, or BAT-40 or at two loci providing one was BAT-26 or c-myb.

Numbers in parentheses are percentages.

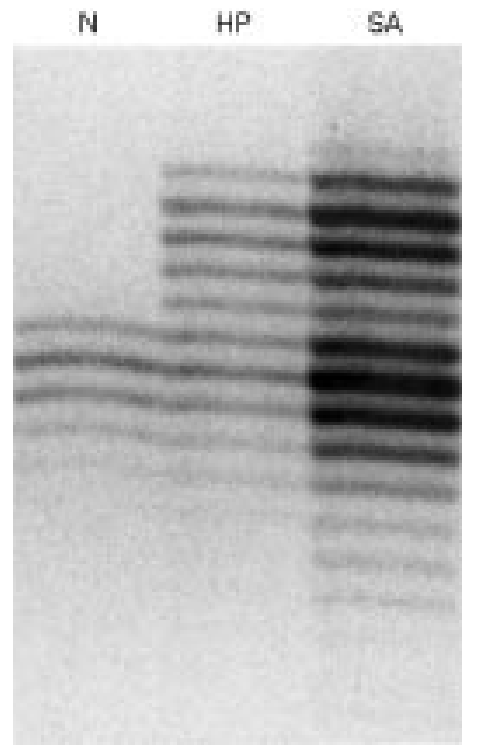

Figure 2 Identical (clonally related) bandshifts are seen for hyperplastic epithelium (HP) and serrated adenoma (SA) at the locus D2S123. DNA was derived from the areas shown in fig $1 . N$, normal DNA obtained from the stalk.

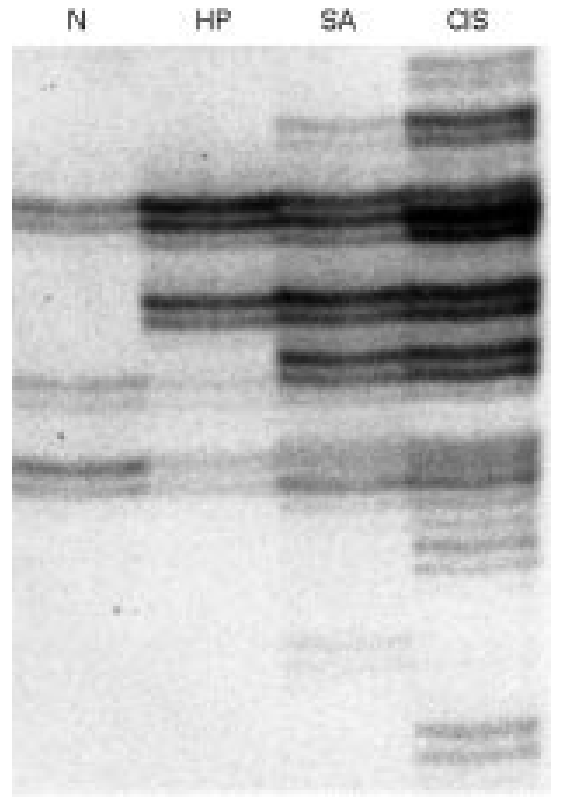

Figure 3 Progressive accumulation of mutations (bandshifts) at the locus MYCL indicative of a stepwise progression from normal to hyperplastic polyp (HP) to serrated adenoma (SA) to carcinoma in situ (CIS).

lium derived from mixed polyps showed MSI, and two were MSI-H. Of the eight mixed polyps containing MSI hyperplastic epithelium, the same mutation was observed within the contiguous neoplastic subclone (serrated or non-serrated adenoma or focus of carcinoma in situ) in four cases (fig 2). In two of these, additional bandshifts were observed in the more advanced subclone that were not evident in the hyperplastic epithelium (fig 3). Of the three cases of MSI-H mixed polyps, one comprised hyperplastic epithelium, serrated adenoma, and severe dysplasia amounting to carcinoma in situ (with a small focus of invasive cancer), one comprised hyperplastic epithelium, serrated adenoma, and tubular adenoma, and the 
Table 4 Frequency of microsatellite instability (MSI) for each locus

\begin{tabular}{lccccccc}
\hline & $\begin{array}{l}\text { No of MSI } \\
\text { samples }\end{array}$ & MYCL & D2S123 & F13B & BAT-40 & BAT-26 & $c-m y b$ \\
\hline Tubular adenoma & 3 & $3(100)$ & $0(0)$ & $0(0)$ & $0(0)$ & $0(0)$ & $0(0)$ \\
Hyperplastic polyp & 6 & $5(83.3)$ & $1(16.7)$ & $0(0)$ & $0(0)$ & $0(0)$ & $0(0)$ \\
Serrated adenoma & 9 & $5(55.6)$ & $4(44.4)$ & $1(11.1)$ & $0(0)$ & $1(11.1)$ & $0(0)$ \\
Mixed polyp & 10 & $6(60.0)$ & $7(70.0)$ & $1(10.0)$ & $3(30.0)$ & $0(0)$ & $2(20.0)$ \\
Total & 28 & $19(67.9)$ & $12(42.9)$ & $2(7.1)$ & $3(10.7)$ & $1(3.6)$ & $2(7.1)$ \\
\hline
\end{tabular}

Numbers in parentheses are percentages.

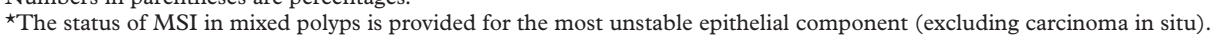

third comprised serrated adenoma and tubular adenoma. Two mixed polyps showed loss of heterozygosity at D2S123 within both hyperplastic epithelium and adjacent adenomatous epithelium. Of the six microsatellite loci tested, 19 of 28 MSI polyps (67.9\%) showed bandshifts at MYCL and 12 of $28(42.9 \%)$ at D2S123 (table 4). These loci were particularly sensitive for the MSI-L phenotype. MSI within hyperplastic polyps showed no significant relation with age, site, size, or morphology (flat $v$ polypoid), but was more common in females (5) than in males $(1)(p=0.0001)$. Pooling serrated adenomas and mixed polyps, MSI was more common in right sided polyps $(p=0.019)$, subjects aged over 60 years $(\mathrm{p}=0.044)$, and possibly in females $(\mathrm{p}=0.063)$.

\section{Discussion}

Between $13 \%$ and $20 \%$ of sporadic colorectal cancers show DNA microsatellite instability. ${ }^{20-24}$ The data relating to sporadic colorectal adenomas are less clear cut. One study showed MSI to be uncommon except in adenomas harbouring foci of severe dysplasia amounting to carcinoma in situ. ${ }^{25}$ By contrast, a recent study showed MSI in 20 of 73 colorectal adenomas $(27 \%){ }^{26}$ Furthermore, that study employed the markers BAT-26 and BAT-40 which are relatively specific for MSI-H. The majority of the adenomas were derived from the proximal colon and 13 of 20 that were MSI-H contained high grade dysplasia (5) or were adjacent to adenocarcinoma (8). ${ }^{26} \mathrm{~A}$ higher frequency of MSI-H has been recorded in adenomas from subjects with hereditary non-polyposis colorectal cancer (HNPCC). ${ }^{27-29}$ In the present study three of 23 tubular adenomas (13\%) were MSI-L and none was MSI-H. On the other hand a relatively high percentage of hyperplastic polyps $(28.6 \%)$ and serrated adenomas $(41.2 \%)$ were MSI-L. The frequency of MSI-L was even higher $(66.7 \%)$ in the hyperplastic epithelium of mixed polyps. The relation with age and MSI is not surprising since most polyps were MSI-L. Only MSI-H neoplasms would be expected to occur at a young age, for example in those with HNPCC. ${ }^{27}$

D2S123 and MYCL were particularly sensitive for the MSI-L phenotype, a finding that fits with data relating to colorectal cancer. ${ }^{23}{ }^{30}$ The division of MSI colorectal cancer into MSI-L and MSI-H is of fundamental importance and this is now widely acknowledged..$^{230-32}$ However, little is known of the molecular or morphological pathogenesis of the MSI-L subset which represents about $10 \%$ of all colorec- tal cancers. Nevertheless, our demonstration of the MSI-L phenotype within a high proportion of hyperplastic polyps and serrated adenomas raises the possibility of a histogenetic relation with MSI-L cancers.

The mixed polyps were especially instructive in this study. The existence of clonal relations between hyperplastic and overtly neoplastic epithelium in four of eight informative cases provides strong evidence that the separate epithelial components comprising mixed polyps are not merely collisions but represent a stepwise continuum. The findings indicate that hyperplastic polyps are not only clonal, but have potential for neoplastic progression even if the realisation of this potential is the exception and not the rule for an individual polyp. Failure to show clonal relations in four mixed polyps could be explained either by collision of independent lesions or diverging molecular evolution. $^{29}$

We do not believe that the findings are explained by inadvertent contamination of the hyperplastic epithelium with DNA originating from the frankly neoplastic subclones. Apart from the fact that microdissection was performed with meticulous care, MSI was observed in $29 \%$ of pure hyperplastic polyps. Furthermore, in two of eight MSI mixed polyps, additional bandshifts were seen in the more advanced subclones imparting a unique DNA fingerprint (fig 3). The absence of these additional bandshifts within the contiguous hyperplastic epithelium serves as evidence of non-contamination. We interpret the progressive accumulation of bandshifts as an epiphenomenon accompanying multistep progression rather than as driving the process, since the loci involved are non-encoding. Nevertheless, the loci MYCL and D2S123 may serve not only as sensitive markers for the MSI-L phenotype, ${ }^{30}$ but also as molecular indicators of hyperplastic polyps that are more likely to progress.

Hyperplastic polyps are normally limited to the sigmoid colon and rectum. Factors predisposing to right sided hyperplastic polyps are the condition hyperplastic polyposis ${ }^{33}$ and female gender. ${ }^{34}$ Interestingly, in this study hyperplastic polyps, serrated adenomas, and mixed polyps were more likely to show MSI in females. Furthermore, MSI polyps that were either mixed or adenomatous were more common in the proximal colon. In a report of a family with hyperplastic polyposis, four affected subjects developed proximal cancers, and the lesions from one of these patients included a hyperplastic polyp and two synchronous cancers with the MSI-L phenotype. ${ }^{10}$ MSI-H cancers (sporadic or in HNPCC) show 
a predilection for proximal colon. Conceivably, some of these may arise from serrated lesions that were initially MSI-L. An early HNPCC cancer has been observed in contiguity with a serrated adenoma in a subject with coincidental hyperplastic polyposis. ${ }^{11}$ Adding these earlier findings to the present study, it is apparent that there is a link between hyperplastic polyps and serrated neoplasia. This sequence is likely to be driven by the mutator phenomenon and to implicate the proximal colon, particularly in females.

In conclusion, the MSI-L phenotype was found in a high proportion of hyperplastic polyps and serrated adenomas. We have suggested that MSL-L hyperplastic polyps may serve as precursors of the subset $(10 \%)$ of colorectal cancers showing the MSI-L phenotype, albeit through the intermediate step of serrated dysplasia. This would be a novel and important morphogenetic pathway for colorectal cancer that differs from the classical "mutator" or MSI-H pathway as well as the "suppressor" or microsatellite stable pathway. A smaller proportion of serrated polyps, particularly right sided lesions, may develop the MSI-H phenotype and progress to MSI-H cancers, both sporadic and in association with HNPCC. However, the majority of MSI-H cancers would not be expected to arise through such a pathway, since the classical adenoma-carcinoma sequence has been well documented in sporadic MSI-H cancers $^{26}$ and in HNPCC. ${ }^{35}$ Finally, although some hyperplastic polyps show neoplastic features at the molecular level, an individual lesion must have very little potential for malignant change. In terms of both their usual location on the crests of mucosal folds and their architecture, hyperplastic polyps appear to arise in part through the mechanism of mucosal prolapse. Clinical concern regarding neoplastic progression should continue to be limited to the condition hyperplastic or serrated adenomatous polyposis. ${ }^{4}{ }^{10}$ The terms "hyperplastic" and the older term "metaplastic" are no longer valid and we therefore suggest that hyperplastic polyps be renamed as serrated polyps since this describes their unique morphology and avoids terms with aggressive clinical connotations that would be unwarranted.

We thank Brenda Mason for typing the manuscript and Lynne Reid for photographic assistance.

1 Vogelstein B, Fearon ER, Hamilton SR, et al. Genetic alterations during colorectal-tumor development. $N$ Engl f Med 1988;319:525-32.

2 Minamoto T, Sawaguchi K, Mai M, et al. Infrequent K-ras activation in superficial-type (flat) colorectal adenomas and activation in superficial-type (flat) colorectal ade
adenocarcinomas. Cancer Res 1994;54:2841-4.

3 Yamagata S, Muto T, Uchida Y, et al. Lower incidence of K-ras codon 12 mutation in flat colorectal adenomas than in polypoid adenomas. Fpn f Cancer Res 1994;85:147-51.

4 Longacre TA, Fenoglio-Preiser CM. Mixed hyperplastic adenomatous polyps/serrated adenomas. A distinct form of colorectal neoplasia. Am $\mathcal{F}$ Surg Pathol 1990;14:524-37.

5 Torlakovic E, Snover DC. Serrated adenomatous polyposis in humans. Gastroenterology 1996;110:748-55.

6 Rubio CA, Kato Y, Hirota T, et al. Flat serrated adenomas of the colorectal mucosa in Japanese patients. In Vivo 1996;10:339-44.

7 Shimamoto F, Tanaka S, Tahara E. Pathogenesis of serrated adenoma of the colorectum: implication for malignant pro-
gression. In: Tahara E, ed. Molecular pathology of gastroengression. In: Tahara E, ed. Molecular pathology of
terological cancer. Tokyo: Springer, 1997:93-106.
8 Ajioka Y, Watanabe $\mathrm{H}$, Jass JR, et al. Infrequent K-ras codon 12 mutation in serrated adenomas of human colorectum. Gut 1998;42:680-4.

9 Otori K, Oda Y, Sugiyama K, et al. High frequency of K-ras mutations in human colorectal hyperplastic polyps. Gut 1997;40:660-3.

10 Jeevaratnam P, Cottier DS, Browett PJ, et al. Familial giant hyperplastic polyposis predisposing to colorectal cancer: A new hereditary bowel cancer syndrome. F Pathol 1996;179: $20-5$

11 Jass JR, Cottier DS, Pokos V, et al. Mixed epithelial polyps in association with hereditary non-polyposis colorectal cancer providing an alternative pathway of cancer histogenesis. Pathology 1997;29:28-33.

12 Konishi M, Kikuchi-Yanoshita R, Tanaka K, et al. Molecular nature of colon tumors in hereditary nonpolyposis colon cancer, familial polyposis, and sporadic colon cancer. Gastroenterology 1996;111:307-17.

13 Lothe RA, Anderson SN, Hofstad B, et al. Deletion of $1 \mathrm{p}$ loci and microsatellite instability in colorectal polyps. Genes Chromosomes Cancer 1995;14:182-8.

14 Bardi G, Pandis N, Fenger C, et al. Deletion of $1 \mathrm{p} 36$ as a primary chromosomal aberration in intestinal tumorigenesis. Cancer Res 1993;53:1895-7.

15 Levi S, Urbano-Ispizua A, Gill R, et al. Multiple K-ras codon 12 mutations in cholangiocarcinomas demonstrated with a sensitive polymerase chain reaction technique. Cancer Res 1991;51:3497-502.

16 Miller SA, Dykes DD, Polesky HF. A simple salting out procedure for extracting DNA from human nucleated cells. Nucleic Acids Res 1988;16:1215.

17 Souza RF, Lei J, Yin J, et al. A transforming growth factor beta 1 receptor type II mutation in ulcerative colitisassociated neoplasms, Gastroenterology 1997;112:40-5.

18 Parsons R, Myeroff LL, Liu B, et al. Microsatellite instability and mutations of the transforming growth factor beta type II receptor gene in colorectal cancer. Cancer Res 1995; 55:5548-50.

19 Peng HZ, Issacson PG, Diss TC, et al. Multiple PCR analyses on trace amounts of DNA extracted from fresh and paraffin wax embedded tissues after random hexamer primer PCR amplification. F Clin Pathol 1994;47:605-8.

20 Aaltonen LA, Peltomäki PS, Leach FS, et al. Clues to the pathogenesis of familial colorectal cancer. Science 1993; 260:812-16.

21 Lothe RA, Peltomäki P, Meling GI, et al. Genomic instability in colorectal cancer: relationship to clinicopathological variables and family history. Cancer Res 1993;53:5849-52.

22 Thibodeau SN, Bren G, Schaid D. Microsatellite instability in cancer of the proximal colon. Science 1993;260:816-19.

23 Jass JR, Do K-A, Simms LA, et al. Morphology of sporadic colorectal cancer with DNA replication errors. Gut 1998;42:673-9.

24 Senba S, Konishi F, Okamoto T, et al. Clinicopathologic and genetic features of nonfamilial colorectal carcinomas with DNA replication errors. Cancer 1998;82:279-85.

25 Young J, Searle J, Buttenshaw R, et al. An Alu VpA marker on chromosome 1 demonstrates that replication errors manifest at the adenoma-carcinoma transition in sporadic colorectal tumours. Genes Chromosomes Cancer 1995;12: 251-4.

26 Grady WM, Rajput A, Myeroff L, et al. Mutation of the type II transforming growth factor- $\beta$ receptor is coincident with the transformation of human colon adenomas to malignant carcinomas. Cancer Res 1998;58:3101-4.

27 Aaltonen LA, Peltomäki P, Mecklin J-P, et al. Replication errors in benign and malignant tumors from hereditary nonpolyposis colorectal cancer patients. Cancer Res 1994; 54:1645-8.

28 Jacoby RF, Marshall SJ, Kailas S, et al. Genetic instability associated with adenoma to carcinoma progression in hereditary nonpolyposis colon cancer. Gastroenterology 1995;109:73-82.

29 Shibata D, Navidi W, Salovaara R, et al. Somatic microsatellite mutations as molecular tumor clocks. Nature Med 1996;2:676-81.

30 Dietmaier W, Wallinger S, Bocker $\mathrm{T}$, et al. Diagnostic microsatellite instability: Definition and correlation with mismatch repair protein expression. Cancer Res 1997;57: 4749-56.

31 Thibodeau SN, French AJ, Cunningham JM, et al. Microsatellite instability in colorectal cancer: different mutator
phenotypes and the principle involvement of hMLH1. phenotypes and the princip
Cancer Res 1998;58:1713-18.

32 Percesepe A, Kristo P, Aaltonen LA, et al. Mismatch repair genes and mononucleotide tracts as mutation targets in colorectal tumors with different degrees of microsatellite instability. Oncogene 1998;17:157-63.

33 Williams GT, Arthur JF, Bussey HJR, et al. Metaplastic polyps and polyposis of the colorectum. Histopathology 1980;4:155-70.

34 Jass JR, Young PJ, Robinson EM. Predictors of presence, multiplicity, size and dysplasia of colorectal adenomas. A necropsy study in New Zealand. Gut 1992;33:1508-14.

35 Jass JR, Stewart SM. Evolution of hereditary non-polyposis colorectal cancer. Gut 1992;33:783-6. 